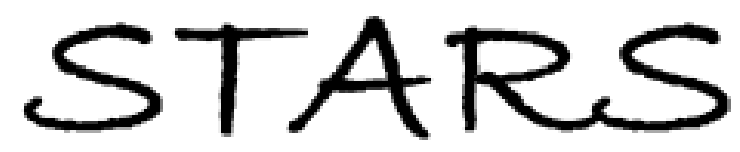

University of Central Florida

STARS

$1-1-2005$

\title{
Precision polarization multiplexed heterodyne acousto-optic interferometric sensor
}

Muzammil A. Arain

University of Central Florida

Nabeel A. Riza

University of Central Florida

Find similar works at: https://stars.library.ucf.edu/facultybib2000 University of Central Florida Libraries http://library.ucf.edu

This Article is brought to you for free and open access by the Faculty Bibliography at STARS. It has been accepted for inclusion in Faculty Bibliography 2000 s by an authorized administrator of STARS. For more information, please contact STARS@ucf.edu.

\section{Recommended Citation}

Arain, Muzammil A. and Riza, Nabeel A., "Precision polarization multiplexed heterodyne acousto-optic interferometric sensor" (2005). Faculty Bibliography 2000s. 4969.

https://stars.library.ucf.edu/facultybib2000/4969

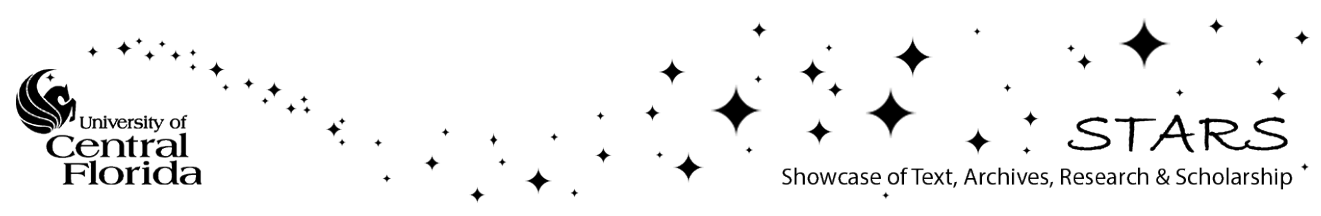




\section{Precision polarization multiplexed heterodyne acousto-optic interferometric sensor}

\author{
Muzammil A. Arain, MEMBER SPIE \\ Nabeel A. Riza, FELLOW SPIE \\ University of Central Florida \\ College of Optics and Photonics/CREOL \\ Photonics Information Processing \\ Laboratory \\ 4000 Central Florida Boulevard \\ Orlando, Florida 32816-2700 \\ E-mail: riza@creol.ucf.edu
}

\begin{abstract}
Proposed and demonstrated is an angstrom level optical path length (OPL) detection sensitivity polarization multiplexed heterodyne acousto-optic interferometric sensor. Because both the phase-coded and the reference rf signals in the design are generated by the same optics, the heterodyne detected rf signals generated contain correlated phase noise characteristics that essentially cancel out during the mixing process in the rf domain. Hence, the proposed four-beam design leads to a super stability OPL sensor with angstrom level experimentally demonstrated measurement stability. A proof-of-concept experiment measures the voltage-dependent phase change due to the refractive index change of a liquid crystal sensor chip. (c) 2005 Society of Photo-Optical Instrumentation Engineers. [DOI: 10.1117/1.1914779]
\end{abstract}

Subject terms: angstrom resolution; heterodyne optical interferometers; acoustooptic devices.

Paper 040539 received Aug. 10, 2004; revised manuscript received Dec. 2, 2004; accepted for publication Dec. 8, 2004; published online May 4, 2005.

\section{Introduction}

Optical interferometry is a useful tool in a number of applications such as thickness measurements, surface structure characterization, and optical sensor applications. ${ }^{1,2} \mathrm{~A}$ number of different types of optical interferometers are available and can be selected for a particular application. ${ }^{3}$ One of the main design issues in optical interferometers is mechanical and thermal instability leading to phase noise in the interferometer output signals. ${ }^{4,5,6}$ To alleviate these issues, a novel acousto-optic heterodyne interferometer was demonstrated that has an in-line design providing internally generated rf reference signal leading to an angstrom optical path length (OPL) resolution. ${ }^{7}$ This interferometer uses a double Bragg cell diffraction approach to measure the phase difference between two optical beams. The most dominant source of noise in an interferometer is phase fluctuations introduced by air currents or temperature differences in the optical path. The interferometer in Ref. 7 has a near common optical path so that the temperature, mechanical, and atmospheric variations affect both the signal and reference beams. Thus at the interferometer output while undergoing heterodyne detection, the unwanted phase variations are cancelled out. This particular feature of the proposed heterodyne scanning interferometer results in a low noise level. In this paper, it is shown how the interferometer design in Ref. 7 can be greatly improved in optical efficiency, power distribution flexibility, and compactness via the use of polarization multiplexing and lens optics. ${ }^{8}$ In addition, a novel front-end optical sensor chip design concept is introduced. The rest of the paper describes the design and related experiments for the proposed interferometer.

\section{Polarization Multiplexed In-Line Heterodyne Interferometer Design}

This section begins with a basic introduction to phase sensitive interferometry, explaining why phase noise reduction is critical for realizing precision sensing. Note that interferometers invariably are used to measure the phase difference between two optical beams. This phase difference can be due to different amounts of distance traveled by the two beams, different refractive indices of the mediums in which the two waves are traveling, or a combination of both refractive index and distance differences. The mentioned phase difference then produces a fringe pattern that can either vary in space or in time. Depending upon the fringe pattern analysis method, interferometers can be broadly divided into two categories. In one type of interferometer, the phase information is encoded in the intensity pattern and this pattern is recorded on large area detectors, holograms, or as images in a CCD camera. The intensity pattern is then processed to give the phase information. The phase information contains phase ambiguity because of wrapping of the phase in the $0-2 \pi$ phase interval. To avoid this ambiguity, rigorous phase unwrapping algorithms are used. ${ }^{9}$ Also these types of interferometers are slower because of the low-speed recording process and the large image data processing requirements. Examples of these types of interferometers are Michelson interferometers, Mach-Zehnder interferometers, and Fizeau interferometers such as those used for surface profilometry and characterization. ${ }^{10,11}$

In the other type of interferometer, the phase difference is measured directly. As the phase of an optical beam cannot be directly measured, the heterodyne process is used to convert optical phase data onto a lower radio frequency (rf). Examples of such heterodyne interferometers are acousto-optic heterodyne interferometers, Zeeman-effect interferometers, and dual frequency interferometers. ${ }^{12-14}$ In all these interferometers, high-speed photodetetors are used to extract optical phase data from the photodetected rf sig-

0091-3286/2005/\$22.00 @ 2005 SPIE 


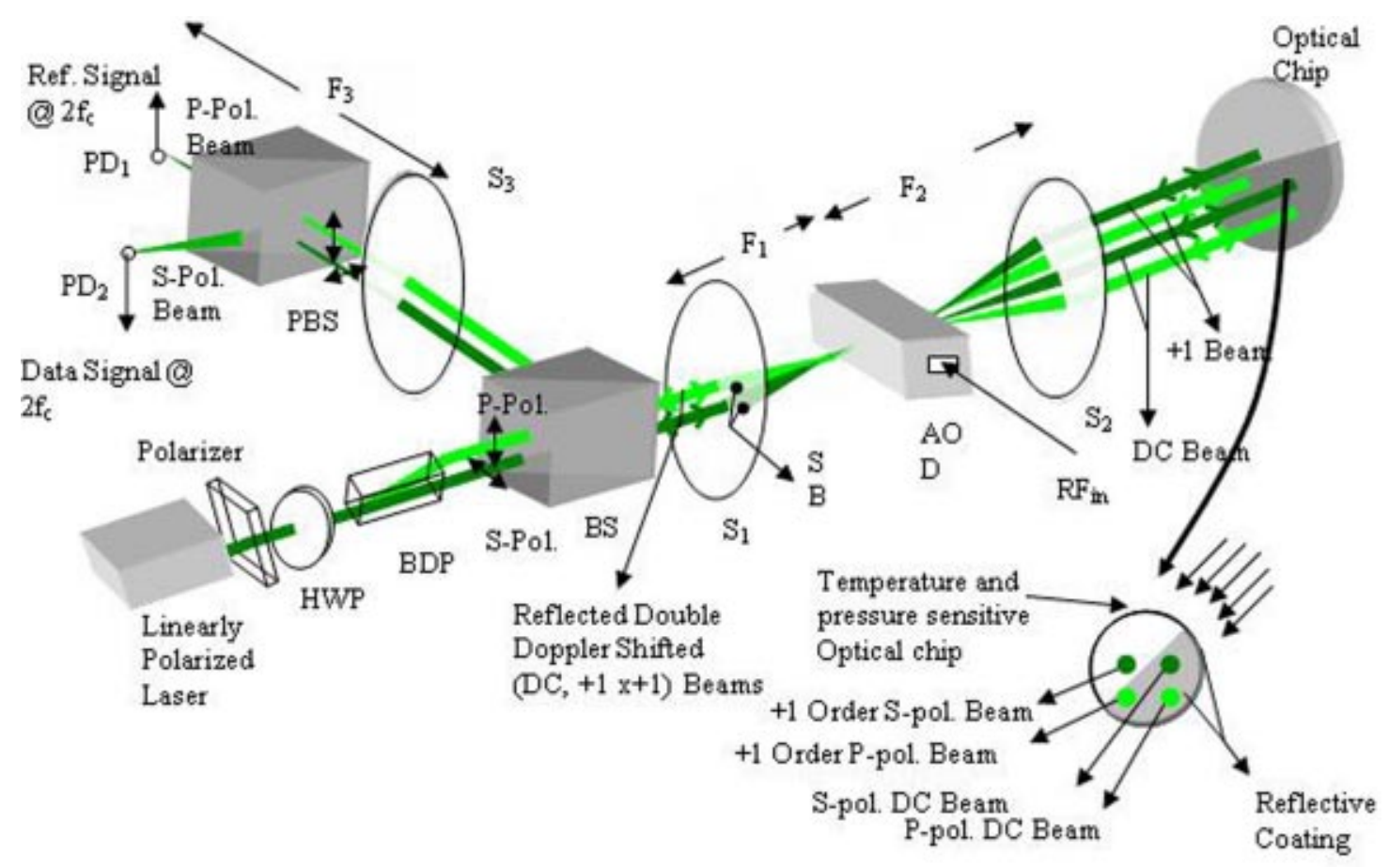

Fig. 1 Schematic diagram of the proposed polarization multiplexed heterodyne scanning interferometer including the novel optical sensor chip for internally referenced phase measurements for high stability. HWP: half wave plate, BDP: beam displacement prism, P-Pol: horizontal polarization, S-Pol: vertical polarization, BS: beam splitter, S: spherical lens, SB: spatial block, AOD: acousto-optic device, PD: photodetector.

nal making these heterodyne interferometers suitable for fast temporal effect sensing applications. A convenient measure of phase difference is the OPL difference between two beams in an interferometer. The OPL between two points $P\left(x_{1}, y_{1}, z_{1}, t_{1}\right)$ and $Q\left(x_{2}, y_{2}, z_{2}, t_{2}\right)$, where $x, y, z$ are the space coordinates and $t$ is the time variable, can be defined as: OPL $=\int_{P}^{Q} n(x, y, z, t) d s$, where $n(x, y, z, t)$ is the refractive index and $d s$ is the distance between the two points. For the simple case when the OPL changes in time but not in space, the OPL can be written as: OPL $=n\left(t_{1}\right)$ $\times d$, where $d$ is the physical path length traveled by the beam and $t_{1}$ is the time instant. The change in OPL at another time $t_{2}$ is then given by: $\Delta \mathrm{OPL}=\left\{n\left(t_{2}\right)-n\left(t_{1}\right)\right\}$ $\times d=\Delta n \times d$, where $\Delta$ represents variations in the associated parameter. If OPL is multiplied with the wave number $K$, the phase change $\Delta \phi$ is obtained. Here $K=2 \pi / \lambda$, where $\lambda$ is the optical wavelength in free-space. The relative phase shift between two beams in the interferometer can then be written as:

$\Delta \phi=(2 \pi / \lambda) \times \Delta \mathrm{OPL}=\frac{2 \pi}{\lambda} \Delta n \times d$.

To measure the interferometer sensed phase $\Delta \phi$ with super accuracy, the sensing rf signal phase noise must be minimized when it is considered against a highly phase stable externally generated $\mathrm{rf}$ reference signal. As reducing phase noise in an interferometer design is extremely difficult due to changing environmental conditions, an alternate approach for measuring the phase difference is to use sensing and reference rf signals generated from the interferometer. In this case, both rf signals have the same or correlated phase noise that can cancel out during the electronic mixing process in the rf phase meter. For example, in general the sensing signal $s(t)$ and reference signal $r(t)$ from a heterodyne interferometer can be written as:

$s(t)=a_{s} \cos \left[2 \pi f_{c} t+\theta_{s}+\phi_{s n}(t)\right]$,

and

$r(t)=a_{r} \cos \left[2 \pi f_{c} t+\theta_{r}+\phi_{r n}(t)\right]$,

where $a, \theta$, and $\phi$ are the amplitude, phase, and phase noise of the respective signals. After electronic mixing and lowpass filtering operation by an rf phase meter, the desired phase meter signal is given by:

$i(t)=\frac{1}{2} a_{r} a_{s} \cos \left[\left(\theta_{s}-\theta_{r}\right)+\left(\phi_{s n}-\phi_{r n}\right)\right]$,

where for example, $\theta_{s^{-}} \theta_{p}=\Delta \phi$, as in Eq. (1). As seen from Eq. (3), for optimal phase meter signal $i(t)$ sensitivity, $\phi_{s n}(t)=\phi_{r n}(t)$. Thus, one approach for getting optimal sensitivity from a heterodyne interferometer is to achieve the $\phi_{s n}(t)=\phi_{r n}(t)$ condition. In a practical implementation, this means designing an optical interferometer such 
that both the sensing signal generating optical beams and the reference generating optical beams suffer the same phase noise due to phase variation generating effects such as vibrations, air current, and temperature fluctuations.

Figure 1 shows how using polarization multiplexing, an optimized interferometer design is realized with the desired common phase noise leading to greatly improved detection efficiency with a minimum of optical components. First a combination of a linear polarizer $\mathrm{P}$ and a laser produces a pure linearly polarized beam. Next a half wave plate (HWP) is used that can change the linear polarization orientation depending upon the orientation of the optic axis of the HWP with respect to the input beam linear polarization. A beam displacement prism (BDP) then divides the input beam into horizontally $(\mathrm{P})$ and vertically $(\mathrm{S})$ polarized beams. The BDP is oriented in such a way that the two beams are displaced vertically from each other. One of the beams acts as a reference beam while the other beam acts as a signal beam. A desired vertical inter-beam displacement can be obtained by proper selection of the length of the BDP crystal. The rotation control of the HWP makes a powerful mechanism to control the relative power in the signal and reference beams. Specifically, if a linear polarizer $\mathrm{P}$ with its axis along the horizontal direction is selected, and the optic axis of the HWP is at $\theta$ with respect to the polarizer axis, the resultant beam is polarized at $2 \theta$ with respect to the horizontal direction. This beam then passes through the BDP, which produces reference and signal beams with normalized powers of $\cos ^{2}(2 \theta)$ and $\sin ^{2}(2 \theta)$, respectively. Hence, any optimum ratio of optical powers between the two beams can be obtained by properly selecting the orientation of the HWP. This in turn provides a power control feature for the reference and signal coded beam in the proposed sensor. The two $\mathrm{S}$ and $\mathrm{P}$ polarized optical beams obtained after the BDP are focused into the AOD or Bragg cell by a spherical lens $S_{1}$ of focal length $F_{1}$ placed at a distance of $F_{1}$ from the Bragg cell at the respective Bragg angle. The AOD is driven by an $\mathrm{rf}$ of $f_{c}$ frequency.

The light output from the AOD consists of two undiffracted dc $\mathrm{P}$ and $\mathrm{S}$ polarized beams of normalized powers of $(1-\eta)$ and two +1 order positive Doppler shifted diffracted $\mathrm{P}$ and $\mathrm{S}$ polarized beams of normalized power $\eta$, where $\eta$ is the diffraction efficiency of the AOD Bragg cell. These dc and +1 order beams then pass through a lens $S_{2}$ of focal length $F_{2}$, which is placed at a distance of $F_{2}$ from the AOD. These beams after passing through the lens become parallel to each other. Note that the four beams coming from $S_{2}$ are all parallel plane waves and hence have a small divergence. Hence, the sensor chip can be placed at a convenient distance for the setup while maintaining the same pass return path for the beams and hence preserving the Bragg condition in the AOD, eliminating the need to place the sensor chip at the focal length. ${ }^{7}$ Note that in the Ref. 7 design, the light output at the sensor plane as well as at the high-speed photodetector plane is a vertical line that reduces the overall optical efficiency of the system. The proposed Fig. 1 design achieves an optically efficient pointto-point design with a minimal number of optical components. This is because the light beams at the sensor chip are small circular beams and the beams at the high-speed de- tectors are focused points thus creating a power efficient system.

The +1 order P-polarized beam produces a signal coded beam that passes through the active zone of the sensor chip and is reflected from the back surface with a reflectivity of $R_{1}$. On the other hand, the P-polarized dc beam and the $\mathrm{S}$-polarized dc, +1 beam pair are reflected back from the highly flat inactive zone of the sensor chip with a reflectivity of $R_{2}$. Thus a variable OPL difference is introduced between the sensing beam pair and the reference beam pair that depends upon the thickness and the refractive index of the active zone of the sensor chip. The material of the sensor chip can be chosen to be sensitive to the measured sensor process variable such as temperature or pressure. Note that S-polarized beam pair ( $\mathrm{dc}$ and +1 order) and the dc P-polarized beam are reflected from the chip front surface and hence do not pass through the sensor chip active layer. These four beams are thus reflected back into the system and are double diffracted on the return path. The +1 order beams produce a double diffracted $(+1,+1)$ order beams that become collinear with their respective dc beams and are stationary. The other pair $(+1,-1)$ order beam are blocked spatially. First a beam splitter (BS) in the return path deflects the light at $90 \mathrm{deg}$ and then a polarization beam splitter (PBS) is used to separate the P-polarized and $\mathrm{S}$-polarized beam pairs. Photodetector $\mathrm{PD}_{1}$ detects the sum of the P-polarized dc with a normalized optical field amplitude of $\left[(1-\eta)^{2} R_{2}\right]^{1 / 2}$ and the double diffracted $(+1 \times+1)$ order beam with a normalized amplitude of $\eta\left(R_{1}\right)^{1 / 2} \cdot \mathrm{PD}_{2}$ detects the sum of the S-polarized dc beam with normalized amplitude of $\left[(1-\eta)^{2} R_{2}\right]^{1 / 2}$ and the double diffracted $(+1$ $X+1)$ order beam with a normalized amplitude of $\eta\left(R_{2}\right)^{1 / 2}$. Both $\mathrm{PD}_{1}$ and $\mathrm{PD}_{2}$ produce heterodyne detected signals at $2 f_{c}$. An efficient heterodyne detection process is implemented when the amplitudes of the respective signals are equal. This condition is achieved in the proposed design by controlling the orientation of the BDP and the HWP in Fig. 1, along with power control of the rf signal applied to the Bragg cell. The signal from $\mathrm{PD}_{1}$ acts as a reference phase signal while the signal from $\mathrm{PD}_{2}$ is the data signal coded with the OPL variation information. Both the reference phase and the test medium phase $2 f_{c}$ frequency signals are fed to an electronic phase meter for sensor parameter measurements. Note that as the rf drive frequency of the AOD is changed to implement one-dimensional scanning of the sensing zone, the dc beams remain stationary while the +1 order P-polarized and S-polarized beams translate linearly by the same physical distance on the chip. Because the reference zone of the chip is highly flat and the +1 order beams travel the same optical path length from the AOD to the chip, the reference phase at the rf phase meter is held constant as needed for processing. In a practical scenario, the reference chip zone can show some flatness variation causing a slight variation in the reference phase as the optical beam is scanned. In this case, as the reference zone optical thickness variation is fixed, one can calibrate the sensor by measuring this reference phase variation and taking it into account for rf phase difference processing. Either way, the proposed sensor can be reference phase noise tolerant. As the sensing parameter changes, the OPL in the sensor chip changes and produces 


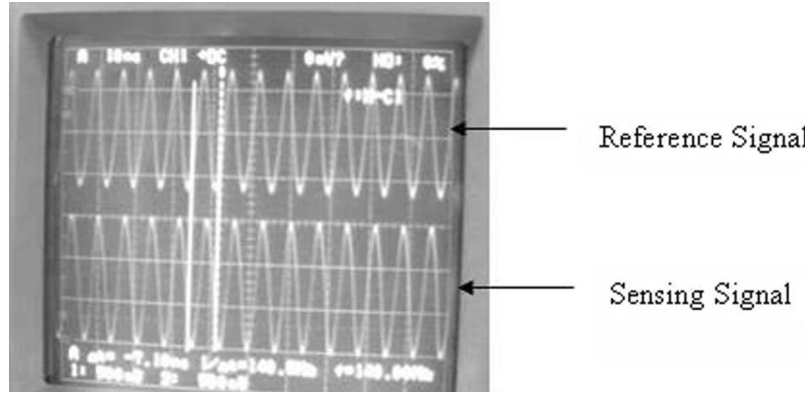

Fig. 2 Oscilloscope traces of the 140-MHz output from the photoreceivers when sensor test material, i.e., a NLC chip, is inserted in the system. The AOD drive is $70-\mathrm{MHz}$. The upper trace is the reference phase data obtained from $\mathrm{PD}_{1}$ while the lower trace is the signal phase obtained from $\mathrm{PD}_{2}$.

a phase difference between the rf signals feeding the phase meter. This phase difference $\Delta \phi$ can directly be calibrated in terms of the sensing parameter, thus realizing a high precision OPL sensor.

In general, the reflectivity of the active zone $R_{1}$ of the sensing chip can be different from the reflectivity $R_{2}$ of the inactive zone of the sensor chip. This will create reference and signal beams of unequal magnitudes and also a nonoptimal heterodyne signal, which hinders optimal phase detection at the phase meter that requires two equal power signals for optimal noise rejection. As mentioned earlier, the orientation of the HWP and the BDP provides the control of optical power in the reference and signal beams. Another important aspect is the different reflectivities of the individual pixels that will create a nonoptimal heterodyne detected rf signals at frequency $2 f_{c}$. This requires power control within the dc and the +1 order beams. This control is provided by the adjusted power of the rf signal applied to the AOD that changes the diffraction efficiency of the AOD in the proposed design. Thus an optimal power split ratio between the dc and the +1 sensing beam can be obtained for the sensing pixel. Hence, the power split control in the reference and signal beam by the orientation of the HWP and the BDP along with the power control of the +1 order beams realizes a sensing system for widely different sensing materials and sensor chips.

\section{Experimental Demonstration}

Vertically or s-polarized light from a $532-\mathrm{nm} 15-\mathrm{mW}$ $\mathrm{Nd}$ :YAG laser passes through a HWP oriented at $22.5 \mathrm{deg}$ to the vertical axis. A BDP is oriented at $45 \mathrm{deg}$ to the horizontal direction producing two vertically displaced equal power beams with a separation of $2 \mathrm{~mm}$. The upper beam is s-polarized while the lower beam is p-polarized. These beams pass through the BS and are focused into an AOD at the respective Bragg angle using a $F_{1}=10 \mathrm{~cm}$ biconvex lens $S_{1}$. The AOD used is a flint glass Bragg cell with a center frequency of $f_{c}=70 \mathrm{MHz}$ and a bandwidth of $40 \mathrm{MHz}$. The active AOD aperture is $39 \mathrm{~mm} \times 2 \mathrm{~mm}$ with an access time of $10 \mu \mathrm{s}$. The AOD is driven by a frequency synthesizer coupled with a power amplifier with a $40 \mathrm{~dB}$ gain.

To demonstrate the validity of the proposed architecture, a 6 - $\mu$ m-thick parallel-rub nematic liquid crystal (NLC)

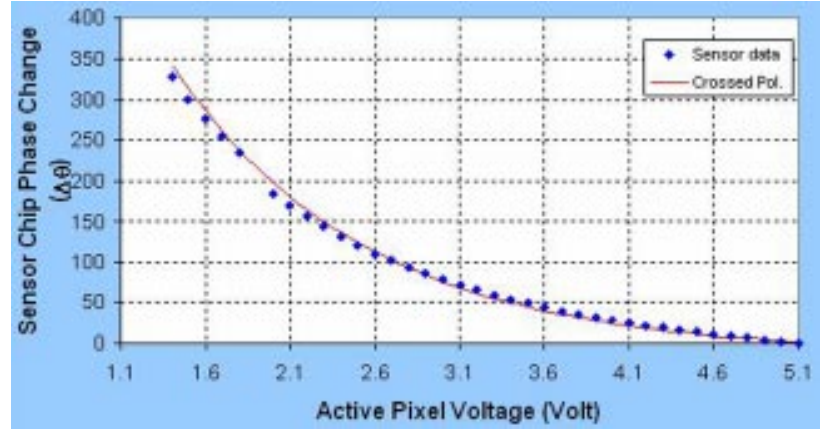

Fig. 3 Experimental data of relative phase shift introduced in the NLC chip in the interferometer by varying the amplitude of the voltage drive signal to the sensing LC pixel.

chip is used to test the instrument. The chip has four identical LC pixels that can be controlled independently by varying a voltage signal to the respective pixel electrodes. The NLC chip is placed with its nematic director along the linear p-polarization of the scanning optical beam. Any amount of optical phase shift between 0 and 330 deg for this particular NLC chip, which corresponds to an optical path length difference of $243.833 \mathrm{~nm}$, can be introduced by varying the amplitude of the $1 \mathrm{KHz}$ square wave voltage drive signal applied to the one active pixel in the NLC chip that corresponds to the +1 order P-polarized beam. Note that the other three LC pixels are not driven by the voltage signal and thus do not introduce any OPL variation. The OPL variation is only introduced in the +1 order p-polarized beam that creates a signal coded beam. Figure 2 shows a oscilloscope trace of the detected $2 f_{c}=140 \mathrm{MHz}$ sensing and reference signals that feed the phase meter. The upper signal is the reference signal while the lower signal is the sensor phase coded signal. The phase angle $\Delta \theta=\theta_{s}$ $-\theta_{r}$ is a function of the LC chip voltage and the LC chip thickness $d$. This phase difference $\Delta \theta$ is measured using a rf lock-in phase meter with an instrument resolution of 0.02 deg. In the experiment a \pm 0.05 deg fluctuation in phase readings is obtained from the lock-in phase meter, which corresponds to experimentally demonstrated resolution of $0.1 \mathrm{deg}$. This in turn sets the OPL measurement resolution of $1.4 \AA$ for the demonstrated sensor. Figure 3 shows the LC sensor chip active pixel phase variations versus the pixel drive voltage. To verify the results, the phase retardation of the NLC chip was measured as a function of applied voltage by measuring the transmitted intensity of the chip when placed between two cross-polarizers with the NLC director of the chip at $45 \mathrm{deg}$ with respect to the polarizer axis. The data is in good agreement with the results obtained by the interferometer measurements.

\section{Conclusion}

In conclusion, we have successfully implemented a compact mode reflective heterodyne scanning optical interferometer that for example can be used to measure thickness changes up to an angstrom sensitivity. The proposed reflective design uses an inline rf and optical design, in addition to using few optical components in the noncommon path scanning arm. This design has inturn led to an interferometer demonstration with exceptionally low phase noise with 
a $1.4 \AA$ OPL measurement resolution. Hence the proposed instrument has the capability to measure path length changes on the atomic scale. It is expected that the demonstrated instrument can be a useful tool for biomedical applications involving molecular and cellular mapping. The instrument resolution can be further increased by using custom compact packaging mechanics and optics.

\section{References}

1. J. Schwider, D. R. Herriot, J. E. Gallagher, D. P. Rosenfeld, A. D. White, and D. J. Brangaccio, "Digital wavefront measuring interferometer for testing optical surfaces and lenses," Appl. Opt. 13, 26932703 (1974)

2. C. Koch, "Measurement of ultrasonic pressure by heterodyne interferometery with a fiber-tip sensor," Appl. Opt. 38, 2812-2819 (1999).

3. M. Born and E. Wolf, Principles of Optics, 7th ed., Cambridge University Press, Oxford (1980)

4. D. N. Wang, Y. N. Ning, A. W. Palmer, K. T. V. Grattan, and K. Weir "An optical scanning technique in a white light interferometric system," IEEE Photonics Technol. Lett. 6, 855-857 (1994).

5. S. Kim, I. Chang, D. Kim, T. Kim, and S. Yoo, "Very large scale phase measuring interferometry for profile measurement of aspheric surfaces with nanometer accuracy," in Pacific Rim Conference on Lasers and Electro-Optics, pp. 70-71, IEEE, New York (1999).

6. J. V. Knuuttila, P. T. Tikka, and M. M. Salomaa, "Scanning Michelson interferometer for imaging surface acoustic wave fields," Opt. Lett. 25, 613-615 (2000)

7. N. A. Riza and M. A. Arain, "Angstrom-range optical path-length measurement with a high-speed scanning heterodyne optical interferometer," Appl. Opt. 42, 2341-2345 (2003).

8. N. A. Riza and M. A. Arain, "Angstrom sensitivity polarization multiplexed heterodyne acousto-optic interferometric sensor," in Third IEEE Sensors Conference, Vienna, Austria, October 24-27, 2004.

9. K. Creath, "Temporal phase measurement methods," in Interferogram Analysis: Digital Fringe Pattern Measurement Techniques, D. W. Robinson and G. T. Reid, Eds., Institute of Physics Publishing Ltd., Bristol, England (1993).

10. T. Suzuki, T. Maki, X. Zhao, and O. Sasaki, "Disturbance-free highspeed sinusoidal phase-modulating laser diode interferometer," Appl. Opt. 41, 1949-1953 (2002).
11. K. Hibino, B. F. Oreb, P. S. Fairman, and J. Burke, "Simultaneous measurement of surface shape and variation in optical thickness of a transparent parallel plate in wavelength-scanning Fizeau interferometer," Appl. Opt. 43, 1241-1249 (2004).

12. N. A. Riza, "Scanning heterodyne optical interferometer," Rev. Sci. Instrum. 67, 2466-2476 (1997)

13. C. Chou, J. Shyu, Y. Huang, and C. Yuan, "Common-path optical heterodyne profilometer: a configuration," Appl. Opt. 37, 4137-4142 (1998).

14. D. Lin, Z. Liu, R. Zhang, J. Yan, C. Yin, and Y. Xu, "Step-height measurement by means of a dual-frequency interferometric confocal microscope," Appl. Opt. 43, 1472-1479 (2004).

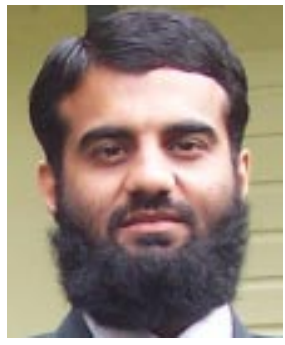

Muzammil Arshad Arain earned his BEngg in electrical engineering from the N.E.D. University of Engineering \& Technology, Karachi, Pakistan. Currently he is a graduate student in the College of Optics/ CREOL at the University of Central Florida, Orlando. He is the recipient of a 2004 SPIE educational scholarship and a 2004 LEOS graduate fellowship.

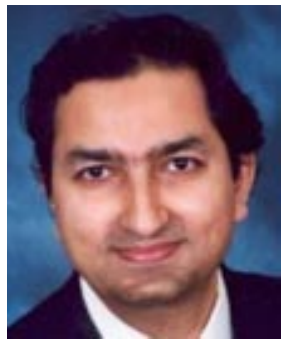

Nabeel A. Riza holds a PhD in electrical engineering from the California Institute of Technology. $\mathrm{He}$ is a fellow of the Optical Society of America (OSA) and the International Society for Optical Engineering (SPIE). After completing his PhD in 1989, Riza joined the General Electric Corporate Research and Development Center. In 1995, he joined College of Optics/CREOL at the University of Central Florida where he is professor of optics. He is also the founder of Nuonics, Inc. He is a 2001 International Commission of Optics (ICO) prize winner. 